\title{
Contribution to harmonic balance calculations of periodic oscillation for self-sustained musical instruments with focus on single-reed instruments
}

\author{
Snorre Farner \\ Department of Electronics and Telecommunications, NTNU, O. S. Bragstads pl. 2, 7491 Trondheim, \\ Norway \\ Christophe Vergez and Jean Kergomard and Aude Lizée \\ Laboratoire de Mécanique et d'Acoustique, CNRS UPR 7051, 31 chemin Joseph Aiguier, 13402 Marseille Cedex 20, \\ France
}

(Dated: November 20, 2018)

\begin{abstract}
The harmonic balance method (HBM) was originally developed for finding periodic solutions of electronical and mechanical systems under a periodic force, but has later been adapted to self-sustained musical instruments. Unlike time-domain methods, this frequency-domain method does not capture transients and so is not adapted for sound synthesis. However, its independence of time makes it very useful for studying every periodic solution of the model, whether stable or unstable without care of initial conditions. A computer program for solving general problems involving nonlinearly coupled exciter and resonator, "Harmbal", has been developed based on the HBM. The method as well as convergence improvements and continuations facilities are thorougly presented and discussed in the present paper. Application of the method is demonstrated on various problems related to a common model of the clarinet: a reed modelled as a simple spring with and without mass and damping, a nonlinear coupling and a cubic simplification of it, and a cylindrical bore with or without dissipation and dispersion as well as a bore formed as a stepped cone.
\end{abstract}

PACS numbers: 43.75.Pq, 43.58.Ta

\section{INTRODUCTION}

Since Helmholtz, ${ }^{?}$ it has become natural to describe a self-sustained ${ }^{1}$ musical instrument as an exciter coupled to a resonator. More recently, McIntyre et al. ${ }^{\text {? have }}$ highlighted that simple models are able to describe the main functioning of most self-sustained musical instruments. These models rely on few equations whose implementation is not CPU-demanding, mainly because the nonlinearity is spatially localized in an area small compared to the wavelength. This makes them well adapted for real-time computation (including both transient and steady states). These models are particularly popular in the framework of sound synthesis.

On the other hand, calculation in the frequency domain is suitable for determining periodic solutions of the model (the values of the harmonics as well as the playing frequency) for a given set of parameters. Such information can be provided by an iterative method named the harmonic balance method (HBM). Though the name "harmonic balance" seems to date back to $1936, ?$ the method was popularized nearly forty years ago for electrical and mechanical engineering purposes, first for forced vibrations, ${ }^{\text {? }}$ later for auto-oscillating systems.? The modern version was presented rather shortly after by Nakhla and Vlach. ${ }^{?}$ In 1978, Schumacher was the first one to use the HBM for musical acoustics purposes with a focus on the clarinet. However in this paper, the playing frequency is not determined by the HBM. This short- coming is the major improvement brought by Gilbert et al. ${ }^{\text {? }}$ eleven years later, who proposed a full study of the clarinet including the playing frequency as an unknown of the problem.

The fact that the HBM can only calculate periodic solutions, may seem as a drawback. Certainly, transients such as the attack are impossible to calculate, and the periodic result is boring to listen to and does not represent the musicality of the instrument. Therefore the HBM is definitely not intended for sound synthesis. Nevertheless, self-sustained musical instruments are usually used to generate harmonic sounds, which are periodic by definition. The HBM is thus very useful to investigate the behavior of a physical model of an instrument, depending on its parameter values. This is possible for both stable and unstable solutions, without care of precise initial conditions. Moreover, HBM results can be compared to approximate analytical calculations (like the variable truncation method (VTM)), ${ }^{\text {? }}$ in order to check the validity of the approximate model considered.

The present paper is based on the work of Gilbert et al. ${ }^{\text {? }}$ Our main contributions are: extension of the diversity of equations managed, improved convergence of the method, introduction of basic continuation facilities, and from a practical point of view, faster calculations.

While the main idea is already described by Gilbert et al., ? Section [II details the principle of the HBM, in particular the discretization of the problem, both in time 
and frequency.

Section [III is devoted to the various contributions of the current work, which are applied in a computer program called Harmbal. ${ }^{\text {? }}$ The framework is defined to include models with three equations: two linear differential equations, written in the frequency domain, and a nonlinear coupling equation in the time domain (see Sec. IIIA). As usual in the HBM, this system of three equations is solved iteratively. The solving method chosen (NewtonRaphson, Sec. IIB has been investigated and its convergence has been improved through a backtracking scheme (Secs. [IIC) and [IID).

To illustrate the advantages of the HBM and the improvements, a few case studies were performed and are presented in Section IV] They are based on a classical model of single reed instruments which is presented in Section IVA In Sections IVB and further, simplifications to each of the three equations are introduced so that the results could be compared to analytical calculations, both for cylindrical and stepped-cones bores. Finally the full model is compared to time-domain simulations. This also shows the modularity of Harmbal. The comparison is achieved through the investigation of bifurcation diagrams as the dimensionless blowing pressure is altered. The derivation of a branch of solution is obtained thanks to basic continuation with an auto-adaptative parameter step.

Finally, various questions are tackled through practical experience from using Harmbal. Section $\nabla$ discusses multiplicity of solutions and poor robustness in the frequency estimation.

\section{NUMERICAL METHOD}

\section{A. The harmonic balance method}

The harmonic balance method is a numerical method to calculate the steady-state spectrum of periodic solutions of a nonlinear dynamical system. In this paper we are only concerned with periodic solutions. The following provides a detailed and general description of the method for a nonlinearly coupled exciter-resonator system.

Let $X\left(\omega_{k}\right), k=0, \ldots, N_{t}-1$ be the Discrete Fourier transform (DFT) of one period $x(t), 0 \leq t<T$, of a $T$-periodic solution of a mathematical system to be defined. $X\left(\omega_{k}\right)$ will have a number of complex components $N_{t}$, which depends on the sampling frequency $f_{s}=1 / T_{s}$ with which we discretize $x(t)$ into $N_{t}=T / T_{s}$ equidistant samples. Furthermore, $\omega_{k}=2 \pi f_{p} T_{s} k$ is the angular frequency of each harmonic of the fundamental frequency $f_{p}$ of the oscillation, referred to as the playing frequency. Note that the sampling frequency $f_{s}=N_{t} f_{p}$ is automatically adjusted to the current playing frequency so that we always consider one period of the oscillation while keeping $N_{t}$ constant. Note also that $N_{t}$ should be sufficiently large to avoid aliasing. Moreover, if it is chosen a power of two, the Fast Fourier transform (FFT) may be used. Assuming that $N_{p}<N_{t} / 2$ harmonics is sufficient to de- scribe the solution, we define $\vec{X} \in \mathbb{R}^{2 N_{p}+2}$ as the $N_{p}+1$ first real components (denoted by $\Re$ ) of $X\left(\omega_{k}\right)$ followed by their imaginary components $(\Im)$ :

$$
\begin{gathered}
\vec{X}=\left[\Re\left(X\left(\omega_{0}\right)\right), \ldots, \Re\left(X\left(\omega_{N_{p}}\right)\right),\right. \\
\left.\Im\left(X\left(\omega_{0}\right)\right), \ldots, \Im\left(X\left(\omega_{N_{p}}\right)\right)\right] .
\end{gathered}
$$

Note that the components $X_{0}$ and $X_{N_{p}+1}$ are the real and imaginary DC components respectively (and that $X_{N_{p}+1}$ is always zero). Our mathematical system can thus be defined by the nonlinear function $F: \mathbb{R}^{2 N_{p}+3} \rightarrow \mathbb{R}^{2 N_{p}+2}$ :

$$
\vec{X}=\vec{F}\left(\vec{X}, f_{p}\right) .
$$

Until now, the playing frequency has silently been assumed to be a known quantity. In autonomous systems, however, the frequency is an additional unknown, so that the $N_{p}$-harmonic solution seeked is defined by $2 N_{p}+3$ unknowns linked through the $2 N_{p}+2$ equations (2). However, it is well known that as $\vec{X}$ is a periodic solution of a dynamical system, any $\vec{X}^{\prime}$ deduced from $\vec{X}$ by a phase rotation (i.e. a shift in the time domain) is also a solution. Thus an additional constraint has to be added in order to select a single periodic solution among the infinity of phase-rotated solutions. A common choice (see Ref.? ) is to consider the solution for which the first harmonic is real (i.e. its imaginary part, $X_{N_{p}+2}$, is zero). This additional constraint decreases the number of unknowns to $2 N_{p}+2$ for an $N_{p}$-harmonic periodic solution. Thus we get $\vec{F}: \mathbb{R}^{2 N+2} \rightarrow \mathbb{R}^{2 N+2}$, and it is now possible to find periodic solutions, if they exist.

Finally, a simple way of avoiding trivial solutions to equation (21) is to look for roots of the function $\vec{G}$ : $\mathbb{R}^{2 N_{p}+2} \rightarrow \mathbb{R}^{2 N_{p}+2}$, defined by

$$
\vec{G}\left(\vec{X}, f_{p}\right)=\frac{\vec{X}-\vec{F}\left(\vec{X}, f_{p}\right)}{X_{1}}
$$

i.e. $\vec{G}\left(\vec{X}, f_{p}\right)=0$. This equation is usually solved numerically through an iteration process, for instance by the Newton-Raphson method as in our case. How to handle the playing frequency $f_{p}$ will be discussed in the following section.

\section{B. Iteration by Newton-Raphson}

The equation $\vec{G}(\vec{X}, f)=0, \vec{G}$ being defined by equation (3), is nonlinear and has usually no analytical solution. (For readability we leave out the index $p$ on the playing frequency until end of Sec. III) This section describes the common, iterative Newton-Raphson method. This is the method used in the program Harmbal (see Section IIII) although it had to be refined with a backtracking procedure to improve its convergence, as discussed in Section

For the sake of later reference, it is useful to recollect the principles of Newton's method for a one-dimensional 


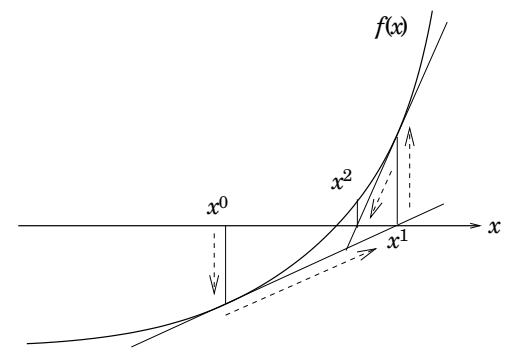

FIG. 1. The iteration process of Newton's method

problem $g(x)=0$. Starting with an estimate $x^{0}$ of the solution, the next estimate $x^{1}$ is defined as the intersection point between the tangent to $g$ at $x_{0}$ and the $x$-axis. The method can be summarized as

$$
x^{i+1}=x^{i}-\frac{g\left(x^{i}\right)}{g^{\prime}\left(x^{i}\right)} .
$$

This is repeated, as shown in Figure 11 while increasing the iteration index $i$ until $g\left(x^{i}\right)<\varepsilon$, where $\varepsilon$ is a userdefined threshold value.

In our $2 N_{p}+2$-dimensional case, we have a vector problem: we search $(\vec{X}, f)$ for which $\vec{G}(\vec{X}, f)=0$. Newton's method is generalized to the Newton-Raphson method, which may be written? ${ }^{\text {: }}$

$$
\left(\vec{X}^{i+1}, f^{i+1}\right)=\left(\vec{X}^{i}, f^{i}\right)-\left(\mathbf{J}_{G}^{i}\right)^{-1} \cdot \vec{G}\left(\vec{X}^{i}, f^{i}\right)
$$

where $\mathbf{J}_{G}^{i} \triangleq \nabla G\left(\vec{X}^{i}, f^{i}\right)$ is the Jacobian matrix of $\vec{G}$ at $\left(\vec{X}^{i}, f^{i}\right)$. Note that all derivatives by $X_{N_{p}+2}$, which was chosen to be zero, are ignored. The column $N_{p}+2$ in the Jacobian is thus replaced by the derivatives with respect to the playing frequency $f . \mathbf{J}_{G}^{i}$ is thus a $\left(2 N_{p}+2\right)$ square matrix. This means that line number $N_{p}+2$ in equation (15) gives the new frequency $f$ instead of $X_{N_{p}+2}$. We define the Newton step $\Delta \vec{X}=\vec{X}^{i+1}-\vec{X}^{i}$ (where $\Delta f=f^{i+1}-f^{i}$ replaces $\Delta X_{N_{p}+2}$ ), which follows the local steepest descent direction.

The Jacobian may be found analytically if $\vec{G}$ is given analytically, but it is usually sufficient to use the firstorder approximation

$$
J_{j k}=\frac{\partial G_{j}}{\partial X_{k}} \simeq \frac{G_{j}\left(\vec{X}+\delta \vec{X}_{k}, f\right)-G_{j}(\vec{X}, f)}{\delta X},
$$

except for $k=N_{p}+2$, in which case we use

$$
J_{j, N+2}=\frac{\partial G_{j}}{\partial f} \simeq \frac{G_{j}(\vec{X}, f+\delta f)-G_{j}(\vec{X}, f)}{\delta f} .
$$

The components of $\delta \vec{X}_{k}$ are zero except for the $k$ th one, which is the tiny perturbation $\delta X$. The iteration has converged when $\left|\vec{G}^{i}\right| \triangleq\left|\vec{G}\left(\vec{X}^{i}, f^{i}\right)\right|<\varepsilon$. We found $\varepsilon=$ $10^{-5}$ to be a good compromize between computation time and solution accuracy.

\section{IMPLEMENTATION AND HARMBAL}

\section{A. Equations for self-sustained musical instruments}

Though, to the authors' knowledge, the harmonic balance method in the context of musical acoustics with unknown playing frequency has only been applied to study models of clarinet-like instruments, it should be possible to consider many different classes of self-sustained instruments. It is well accepted that sound production by a musical instrument results from the interaction between an exciter and a resonator through a nonlinear coupling. Moreover, in most playing conditions, linear modelling of both the exciter and the resonator is a good approximation.

Therefore, within these hypotheses, any musical instrument could be modelled by the following three equations:

$$
\left\{\begin{array}{c}
Z_{e}(\omega) X_{e}(\omega)=X_{c}(\omega) \\
X_{c}(\omega)=Z_{r}(\omega) X_{r}(\omega) \\
\mathcal{F}\left(x_{c}(t), x_{e}(t), x_{r}(t)\right)=0
\end{array}\right.
$$

where $Z_{e}$ is the dynamic stiffness and $Z_{r}$ is the input impedance of the exciter and the resonator, respectively, and $X_{e}$ and $X_{r}$ are the spectra describing the dynamics of the exciter and the resonator during the steady state (periodicity assumption). $X_{c}$ is the spectrum of the coupling variable. All these quantities, and thus equations 8 b), are defined in the Fourier domain. Equation (8) is written in the time domain, where $\mathcal{F}$ is a nonlinear functional of $x_{c}, x_{e}$, and $x_{r}$, which are the inverse Fourier transforms of $X_{c}, X_{e}$, and $X_{r}$, respectively. We apply the discretization as described in Section ПA implying that equations $(8 \mathrm{a}-\mathrm{b})$ become vector equations where the impedances must be written as real $\left(2 N_{p}+2\right) \times\left(2 N_{p}+2\right)$ matrices to accommodate the rules of complex multiplication:

$$
Z(f)=\left(\begin{array}{cc}
\Re(\tilde{Z}(f)) & -\Im(\tilde{Z}(f)) \\
\Im(\tilde{Z}(f)) & \Re(\tilde{Z}(f))
\end{array}\right)
$$

where

$$
\tilde{Z}(f)=\left(\begin{array}{cccc}
Z(0) & 0 & \cdots & 0 \\
0 & Z\left(\omega_{1}\right) & & 0 \\
\vdots & & \ddots & \vdots \\
0 & 0 & \cdots & Z\left(\omega_{N_{p}}\right)
\end{array}\right)
$$

is complex, and $\Re(\tilde{Z})$ and $\Im(\tilde{Z})$ are the real and imaginary components of $\tilde{Z}$. The system (8) is solved iteratively by Harmbal according to the scheme illustrated in Figure 2]

In Harmbal, these equations are easily defined by writing new $\mathrm{C}$ functions. Only superficial knowledge of the $\mathrm{C}$ language is necessary to do this.

Three cases related to models of single reed instruments with cylindrical or stepped-conical bores are studied in particular in Section IV in order to validate the code and to illustrate the modularity of Harmbal. 


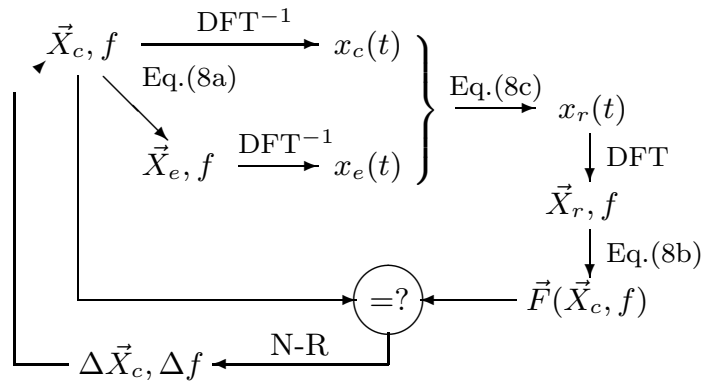

FIG. 2. The iteration loop of the harmonic balance method for a musical instrument (notations defined in the text)

\section{B. Practical characteristics of Harmbal}

Both fast calculation, good portability, and independence of commercial software are easily achieved by programming in $\mathrm{C}$, whose compiler is freely available for most computer platforms. It is, however, somewhat difficult to combine portability with easy usage, because an intuitive usage normally means a graphical and interactive user interface, while the handling of graphics varies a lot between the different platforms.

We have chosen to write Harmbal with a nongraphical and non-interactive ${ }^{2}$ user interface. The major advantage of this is that independent user interfaces may be further developed depending on need.

Our concept is to save both the parameters and the solution in a single file. This file also serves as input to Harmbal while individual parameters can be changed through start-up arguments. The solution provided by the file works as the initial condition for the harmonic balance method. Thus the lack of a simple user interface is compensated by a simple way of re-using an existing solution to solve the system for a slightly different set of parameters. Solutions for a range of a parameter values may thereby be calculated by changing the parameter stepwise and providing the previous solution as an initial condition for the next run. The Perl script hbmap provides such zeroth-order continuation facilities. This procedure may also be used when searching for a solution where it is difficult to provide a sufficiently good initial condition, for instance by successively increasing $N_{p}$ when wanting many harmonics.

\section{Convergence of Newton-Raphson}

When merely employing the Newton-Raphson method to determine the solution of the system at a given set of parameters, we have found that it is impossible to find a solution at particular combinations of the parameters. Indeed, for the clarinet model of Section IVB 1. no convergence was obtained for particular values of the parameter $\gamma$ (the dimensionless blowing pressure) and its neighborhood. This is seen as discontinuities, or holes, in the curves in Figure 3 (see Section [IV] for the underlying equations and parameters). Note that the solu-

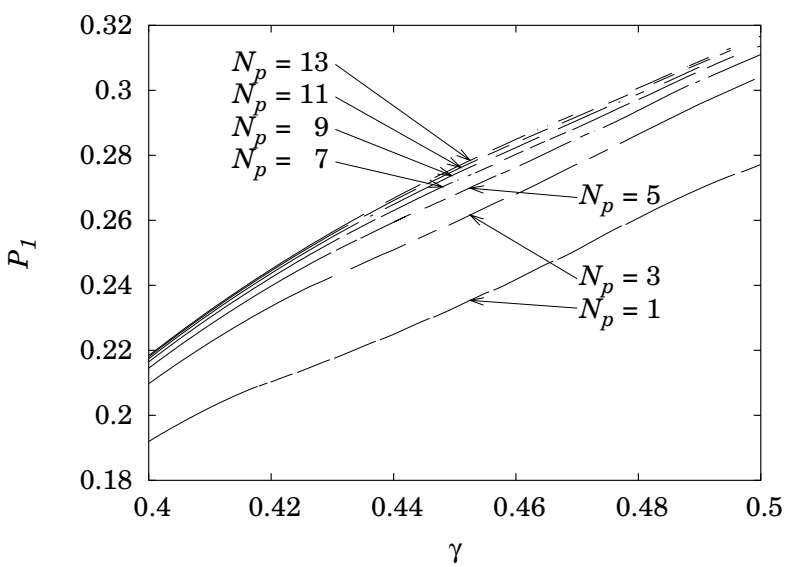

FIG. 3. Solution holes: first pressure harmonic $P_{1}$ versus blowing pressure $\gamma$ for different $N_{p}$ with $N_{t}=128, \zeta=0.5$, and $\eta=10^{-3}$. (Even $N_{p}$ give the same as $N_{p}-1$.) Equations and parameters are defined in section IV]

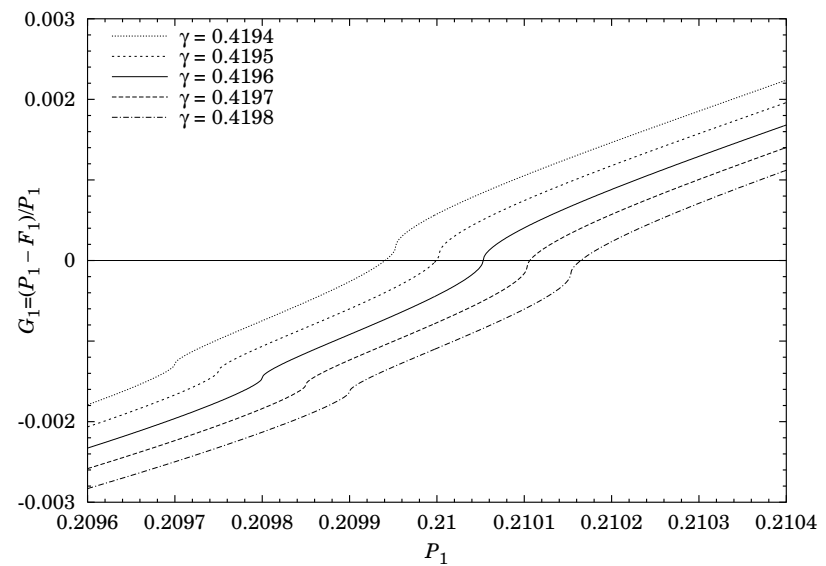

FIG. 4. $G_{1}$ as $P_{1}$ varies around the solution $G_{1}=0$ for various $\gamma$ around a hole at $\gamma \simeq 0.4196$. $N_{t}=128$ and $N_{p}=1$.

tions seem to go continuously through this hole and that the positions of the holes and their extent vary with the number of harmonics $N_{p}$ taken into account. The curves were calculated by the program hbmap. In this case we have decreased $\gamma$ from 0.5 downward in steps of $10^{-4}$ and drawn a line between them except across $\gamma$ values where solution failed. In the holes, the Newton-Raphson method did not converge, either by alternating between two values of $\vec{P}$ (i.e. $\vec{X}_{c}$ ) or by starting to diverge.

To study the problem, we simplified the system to a one-dimensional problem by setting $N_{p}=1$, thus leaving $P_{1}$ as the only nonzero value. $G_{1}$ thus became the only contributor to $|\vec{G}|$, and a simple graph of $G_{1}$ around the solution $G_{1}=0$ could illustrate the problem, as shown in Figure 4 We see that the curve of $G_{1}\left(P_{1}\right)$ has inflection points (visible as "soft steps" on the curve) at rather regular distances. At the centre of a convergence hole, i.e. for $\gamma \simeq 0.4196$, an inflection point is located at the intersection with the horizontal axis. This is a school 
example of a situation where Newton's method does not converge because the Newton step $\Delta P_{1}$ brings us alternatingly from one side of the solution to the other, but not closer.

In fact, the existence of inflection points is linked with the digital sampling of the continuous signal. If the sampling rate is increased, i.e. if $N_{t}$ is increased, the steps become smaller but occur more frequently, as shown for $N_{t}=32,128$, and 1024 in Figures 5 a - c. The derivative $d G_{1} / d P_{1}$ is included in the figures to quantify the importance of the steps. According to the Figures $5 \mathrm{a}-\mathrm{c}$ it seems reasonable to increase $N_{t}$ to avoid convergence problems. However, this would significantly increase the computational cost. Another solution is therefore suggested in the following.

\section{Backtracking}

When the Newton-Raphson scheme fails to converge, it often happens because the Newton step $\Delta \vec{X}$ leads to a point where $|\vec{G}(\vec{X}, f)|$ is larger than in the previous step. However, acknowledging that the Newton step points in the direction of the steepest descent, there must be a point along $\Delta \vec{X}$ where $|\vec{G}(\vec{X}, f)|$ is smaller than in the previous iteration of the HBM. A backtracking algorithm described in Numerical Recipes? (Sec.9.7) solves the problem elegantly by shortening the Newton step as described here. The principle is illustrated in the simple one-dimensional case in Figure 6] where $g(x)$ replaces $|\vec{G}(\vec{X}, f)|$, although we use the multidimensional notation in the following. Defining the $\lambda$ axis along the Newton step, we simply take a step $\lambda \Delta \vec{X}$ in the same direction, where $0<\lambda<1$. The optimal value for $\lambda$ is the one that minimizes the function $h(\lambda)$ :

$$
h(\lambda)=\frac{1}{2}\left|\vec{G}\left(\vec{X}^{i}+\lambda \Delta \vec{X}\right)\right|^{2}
$$

with derivative

$$
h^{\prime}(\lambda)=\left.\left(\mathbf{J}_{G} \cdot \vec{G}\right)\right|_{\vec{X}^{i}+\lambda \Delta \vec{X}} \cdot \Delta \vec{X} .
$$

During the calculation of the failing Newton step, we computed $\vec{G}\left(\vec{X}^{i}\right)$ and $\vec{G}\left(\vec{X}^{i+1}\right)$, so now it is possible to calculate with nearly no additional computational effort $h(0)=\frac{1}{2}\left|\vec{G}\left(\vec{X}^{i}\right)\right|^{2}, h^{\prime}(0)=-\left|\vec{G}\left(\vec{X}^{i}\right)\right|^{2}$, and $h(1)=$ $\frac{1}{2}\left|\vec{G}\left(\vec{X}^{i}+\Delta \vec{X}\right)\right|^{2}=\frac{1}{2}\left|\vec{G}\left(\vec{X}^{i+1}\right)\right|^{2}$. This allows to propose a quadratic approximation of $h$ for $\lambda$ between 0 and 1, for which the minimum is located at

$$
\lambda_{1}=-\frac{\frac{1}{2} h^{\prime}(0)}{h(1)-h(0)-h^{\prime}(0)} .
$$

It can be shown that $\lambda_{1}$ should not exceed 0.5 , and in practice $\lambda_{1} \geq 0.1$ is required to avoid a too short step at this stage.

If $\left|\vec{G}\left(\vec{X}^{i}+\lambda_{1} \Delta \vec{X}\right)\right|$ still is larger than $\left|\vec{G}\left(\vec{X}^{i}\right)\right|, h(\lambda)$ is then modelled as a cubic function (using $h\left(\lambda_{1}\right)$ which has just been calculated). The minimum of this cubic function gives a new value $\lambda_{2}$, again restricted to $0.1 \lambda_{1}<\lambda_{2}<0.5 \lambda_{1}$. This calculation requires solving a system of two equations, so if also $\lambda_{2}$ is not accepted because $\left|\vec{G}\left(\vec{X}^{i}+\lambda_{2} \Delta \vec{X}\right)\right|$ is still too large, we do not enhance to a fourth-order model of $h$, which would increase the computational cost much more. Instead, subsequent cubic modellings are performed using the most two recent values of $\lambda$. In practice, however, not many repetitions should be necessary before finding a better solution, if possible.

\section{CASE STUDIES}

\section{A. The equations for the clarinet}

The three equations $(8 \mathrm{a}-\mathrm{c})$ may be constructed by physical modelling. In the case of the clarinet, a common simple model is described below. We limit the description in the following to a brief presentation based on dimensionless quantities, dimensional variables being denoted by a hat $\left(^{\wedge}\right)$ hereafter (see Fritz et al. ${ }^{?}$ for further details).

The exciter is an oscillating reed which may be modelled as a spring with mass and damping:

$$
\ddot{\hat{y}}+g_{e} \dot{\hat{y}}+\omega_{e}^{2} \hat{y}=\frac{1}{\mu_{e}}\left(\hat{p}-p_{m}\right),
$$

where $\hat{y}$ is the dynamic reed displacement, and $\hat{p}$ and $p_{m}$ are the dynamic pressure in the mouthpiece, i.e. the internal pressure, and the static blowing pressure in the player's mouth, respectively. The constants $\mu_{e}, g_{e}$, and $\omega_{e}$ represent the mass per area, the damping factor, and the angular resonance frequency of the exciter (the reed). The dots over $\hat{y}$ denote the time derivative. In dimensionless form, equation (14) becomes

$$
M \ddot{x}+R \dot{x}+K x=p,
$$

where $p=\hat{p} / p_{M}$ and $x=\hat{y} / H+\gamma / K$ with $\gamma=p_{m} / p_{M}$. The equilibrium reed opening is $H$ as shown in Figure 7. In the static regime, when blowing harder than a maximum pressure $p_{M}$, i.e. $p_{m} \geq p_{M}(\gamma \geq 1)$, the reed blocks the opening, i.e. $\hat{y}=-H$, so we get $\hat{p}=0$ and can conclude that $K=1$ for the current reed model.

Like Fritz et al. ${ }^{?}$ we relate the dimensionless time to the resonance angular frequency $\omega_{r}$ of the resonator (the bore), i.e. $t=\omega_{r} \hat{t}$, so that the values of the dimensionless mass $M$, damping $R$, and spring constant $K$ become

$$
\begin{aligned}
& K=\mu_{e} H \omega_{e}^{2} / p_{M}=1, \\
& R=K g_{e} \omega_{r} / \omega_{e}^{2}=g_{e} \omega_{r} / \omega_{e}^{2} \\
& M=K \omega_{r}^{2} / \omega_{e}^{2}=\omega_{r}^{2} / \omega_{e}^{2} .
\end{aligned}
$$

In the Fourier domain, Equation (15) thus takes the form of equation (8), $Z_{e}(\omega) X(\omega)=P(\omega)$, where

$$
Z_{e}(\omega)=1-M\left(\frac{\omega}{2 \pi}\right)^{2}+i R\left(\frac{\omega}{2 \pi}\right)
$$



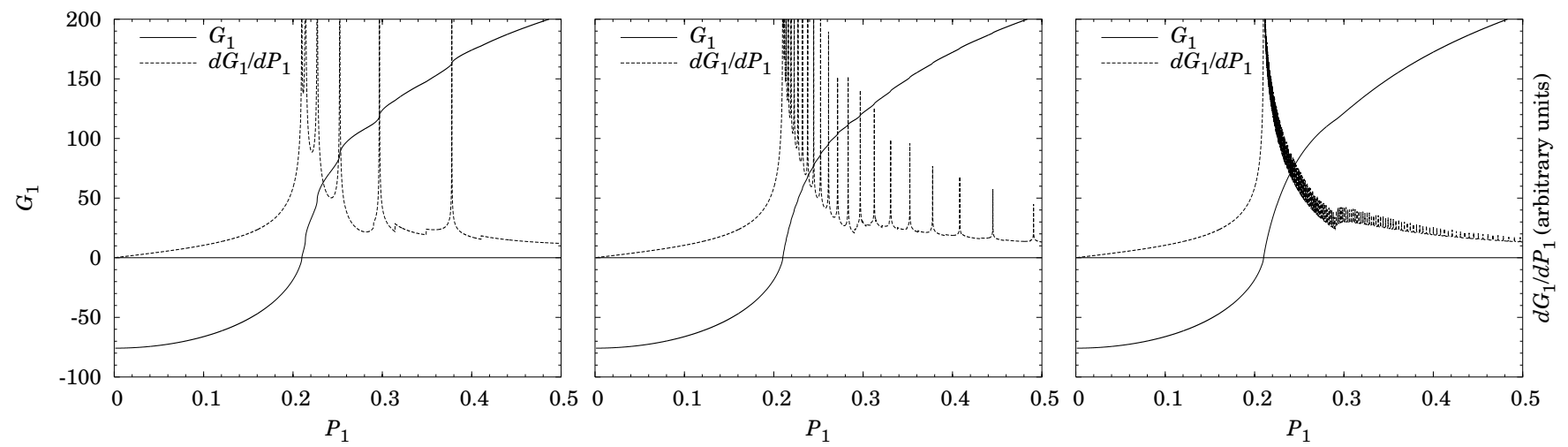

FIG. 5. The effect of sampling rate on the "smoothness" of $G_{1}\left(P_{1}\right)$ : (a) $N_{t}=32$, (b) 128 , and (c) 1024 . The derivative $d G_{1} / d P_{1}$ exhibits the "roughness".

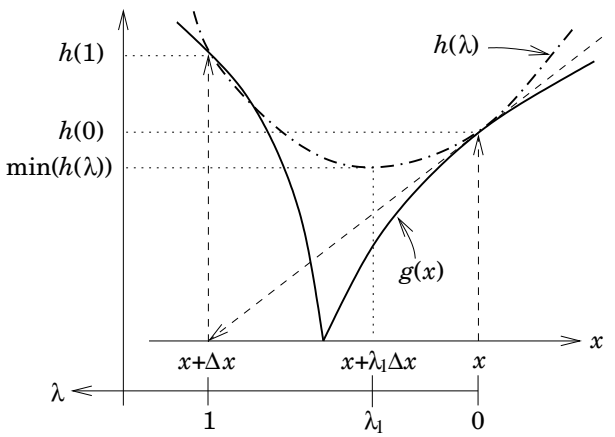

FIG. 6. The principle of backtracking in one dimension. Objective is to estimate the root of $g(x)$ (solid curve). Broken lines with arrows show how the Newton step $\Delta x$ from $x$ leads to divergence. $h(\lambda)$ (dot-dashed curve) is a 2nd order expansion of $g(x)$ along the Newton step, i.e. the $\lambda$ axis. Minimum of $h(\lambda)$ should be closer to the root of $g(x)$ than $g(x+\Delta x)$.

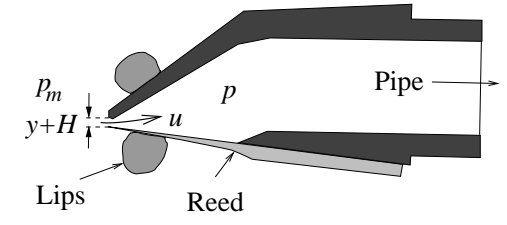

FIG. 7. Illustration of the mouthpiece

for $i=\sqrt{-1}$ and $\omega=2 \pi \hat{\omega} / \omega_{r}=\hat{\omega} / f_{r}$ is the dimensionless angular frequency in the Fourier domain.

A common minimum model for the clarinet assumes a simple reed with no mass or damping, thus $M=R=0$. Equation (15) reduces to $x=p$.

The resonator (i.e. the air column in the bore of the instrument) is commonly described by its frequency response $\hat{Z}_{r}(\hat{\omega})$. For a simple cylindrical bore of length $l$ with a closed and an ideal open end, the resonance frequencies are odd multiples of $f_{r}=c / 4 l, c$ being the sound speed in the air column.? The input impedance of the bore may thus be expressed in dimensionless quantities as

$$
Z_{r}(\omega)=\frac{\hat{Z}_{r}(\hat{\omega})}{Z_{0}}=i \tan \left(\frac{\omega}{4}+(1-i) \alpha(\omega)\right),
$$

where $\alpha(\omega) \triangleq \psi \eta \sqrt{\omega / 2 \pi}$ with $\psi \simeq 1.3$ for common conditions in air and $\eta$ being the dimensionless loss parameter, which depends on the tube length, typically 0.02 for a normal clarinet with all holes closed. $Z_{0} \triangleq \rho c / S$ is the characteristic impedance of the cylidrical resonator, $S$ being its cross section, and $\rho$ the density of air. The last term in the argument of equation (20) includes the dispersion as the real part and viscous losses as the imaginary part.

Equation (8b) becomes

$$
P(\omega)=Z_{r}(\omega) U(\omega),
$$

where $P(\omega)$ and $U(\omega) \triangleq \hat{U}(\omega) Z_{0} / p_{M}$ are the dimensionless internal pressure and volume flow of air through the mouthpiece in the Fourier domain.

The coupling equation (8:), is given by the Bernoulli theorem with some supplementary hypotheses applied between the mouth and the outlet of the reed channel. The coupling equation is nonlinear and must be calculated in the time domain. This leads to the following expression for the dimensionless airflow through the mouthpiece? :

$$
u(p, x)=\zeta(1+x-\gamma) \sqrt{|\gamma-p|} \operatorname{sign}(\gamma-p)
$$

as long as $x>\gamma-1$, and $u=0$ otherwise. $\zeta=$ $Z_{0} w H \sqrt{2 / \rho p_{M}}$ is a dimensionless embouchure parameter roughly describing the mouthpiece and the position of the player's mouth, $w$ being the width of the opening and $\rho$ the density of the air. $\zeta$ is also related to the maximum volume velocity entering the tube.

If the reed dynamics were not taken into account, we had $x=p$ and thus

$$
u(p)=\zeta(1+p-\gamma) \sqrt{|\gamma-p|} \operatorname{sign}(\gamma-p)
$$

for $p>\gamma-1$, and, as before, $u=0$ otherwise. 


\section{B. Verification of method and models}

In the following we want to verify that the HBM (and its implementation in Harmbal) gives correct results. By using very low losses in the resonator (small $\eta$ ) we can compare the results of the HBM with analytical results. Rising the attenuation in the resonator and including mass and damping for the exciter, we compare with numerical results from real-time synthesis of the same system. This also gives us the opportunity to illustrate the modularity of Harmbal as we change the models of the resonator and the nonlinear coupling.

\section{Helmholtz oscillation for cylindrical tubes}

To compare the HBM results with analytical results, we assume a nondissipative, nondispersive air column, i.e. setting $\eta=0$ and thus $\alpha=0$ in equation (20). Furthermore, we assume that the reed has neither mass nor damping and thus use equation (23). The resulting square-wave amplitude (the Helmholtz motion)? may be found by solving $u(p)=u(-p)$, which results from the fact that the internal pressure $p(t)$ and the power $p(t) u(t)$ averaged over a period are zero according to the lossless

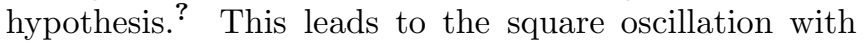
amplitude

$$
p(\gamma)=\sqrt{-3 \gamma^{2}+4 \gamma-1} .
$$

This result is compared with the results calculated by Harmbal (for the same set of equations, but $\eta=10^{-5}$ instead of $\eta=0$ to avoid infinite impedance peaks) for 3, 9, 49, and 299 harmonics close to the oscillation threshold in Figure 8 and at $\gamma=0.4$ in Figure 9 which is far from the threshold.

As expected, the solution using the HBM shows good convergence towards the Helmholtz motion as the number of harmonics increases. Note the deviation for higher harmonics close to the threshold, even for 299 harmonics. Dissipation in the resonator $\left(\eta=10^{-5} \neq 0\right)$ causes higher harmonics to be damped more in this area of $\gamma$ than for higher blowing pressures (as explained e.g. in Ref.? ). The deviation from a square-wave signal is thus more noticeable close to the threshold, and as the HBM calculations imposed a nonzero dissipation, this is probably the reason for the small deviation in Figure 8 The deviation is not visible in the time domain.

A popular simplification of the nonlinear function (23) is a cubic expansion for small oscillations (e.g. Ref.) ? ? ?

$$
\tilde{u}(p)=u_{00}+A p+B p^{2}+C p^{3},
$$

where $u_{00}, A, B$, and $C$ are easily found by expanding equation (23). Its Helmholtz solution is easily calculated like above, yielding

$$
p(\gamma)=\sqrt{-\frac{A}{C}}=\sqrt{\frac{8 \gamma^{2}(3 \gamma-1)}{\gamma+1}} .
$$
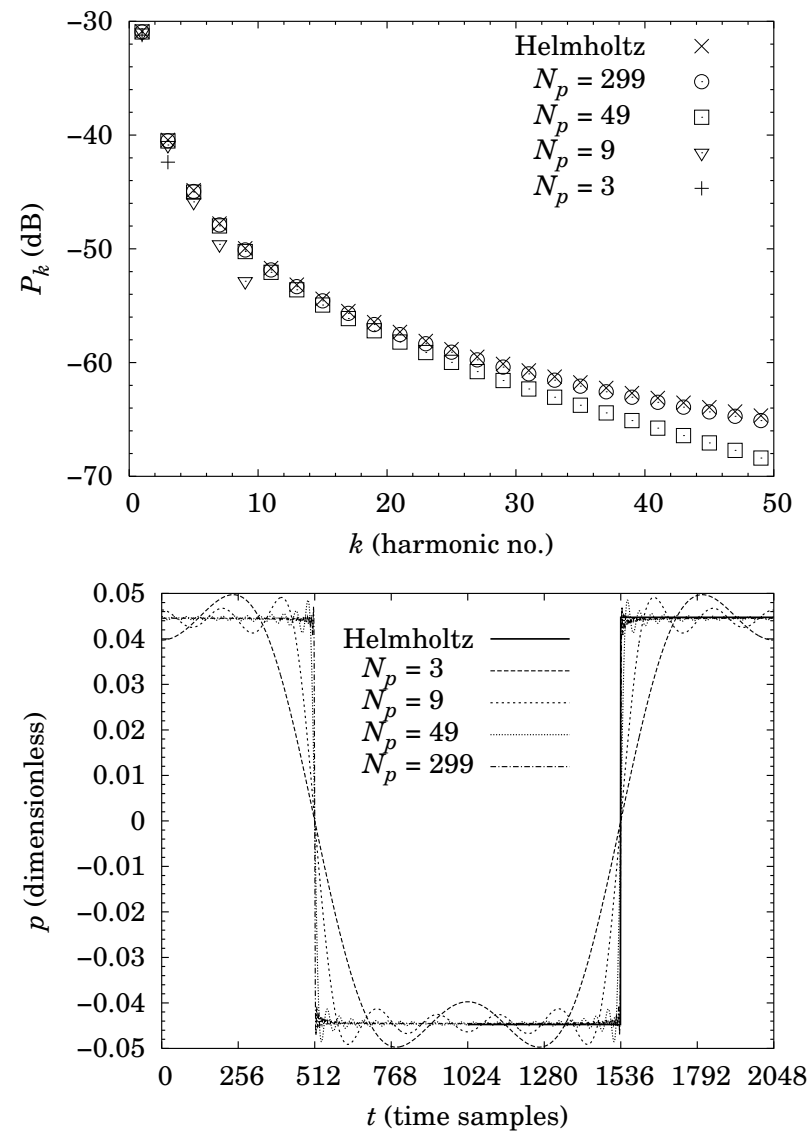

FIG. 8. The Helmholtz solution, eq. (24) compared with the HBM truncated to 3, 9, 49, and 299 harmonics close to the oscillation threshold $\left(\gamma=0.334, \zeta=0.5, \eta=10^{-5}\right)$. (a) frequency domain. (b) time domain.

The influence of the difference between the two versions of the nonlinear function is investigated in Figure [10 for the lossless case. Close to the oscillation threshold, Figure 10k, we see that there is no significant difference between the two versions of the nonlinear equation, as expected. The fact that the HBM is lower for higher harmonics is as before due to the small attenuation we had to include to perform the numerical calculations. Far from the threshold, however, Figure 10b, we see that the cubic expansion fails to approximate the nonlinear equation. For lower harmonics this error is larger than the attenuation effect in the HBM calculations. This is further discussed by Fritz et al. ?

In Figure 11] we have completed some of the curves that we failed to make in Figure 3] and even increased the number of harmonics, owing to the backtracking mechanism. Admittedly, at $N_{p}=49$, a few holes can still be seen, but the convergence is significantly improved.

Here the amplitude of the first harmonic is plotted for different numbers of harmonics as a function of the blowing pressure $\gamma$ together with first harmonic of the Helmholtz solution, deduced from equation (24). In practice, the solution at $\gamma=0.4$ was found and then hbmap 


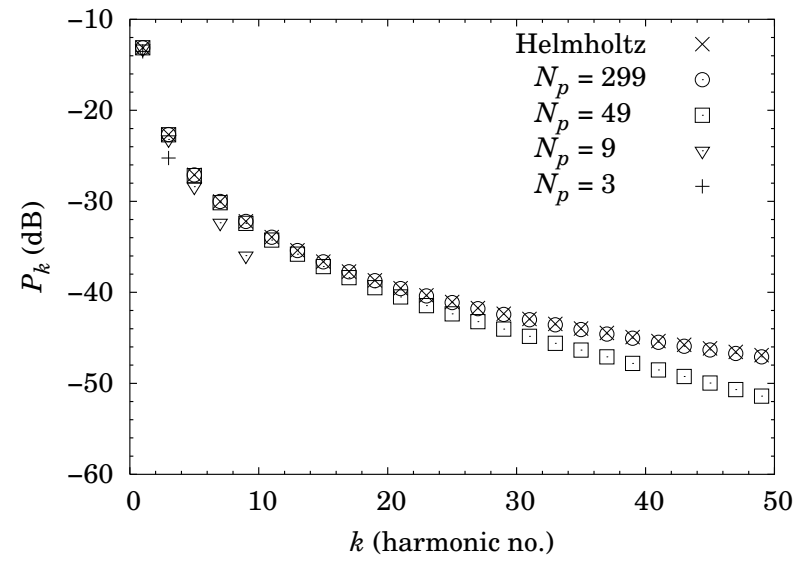

FIG. 9. The Helmholtz solution, eq. (24) compared with the HBM truncated to 3, 9, 49, and 299 harmonics far from the oscillation threshold $\left(\gamma=0.40, \zeta=0.5, \eta=10^{-5}\right)$ in the frequency domain.
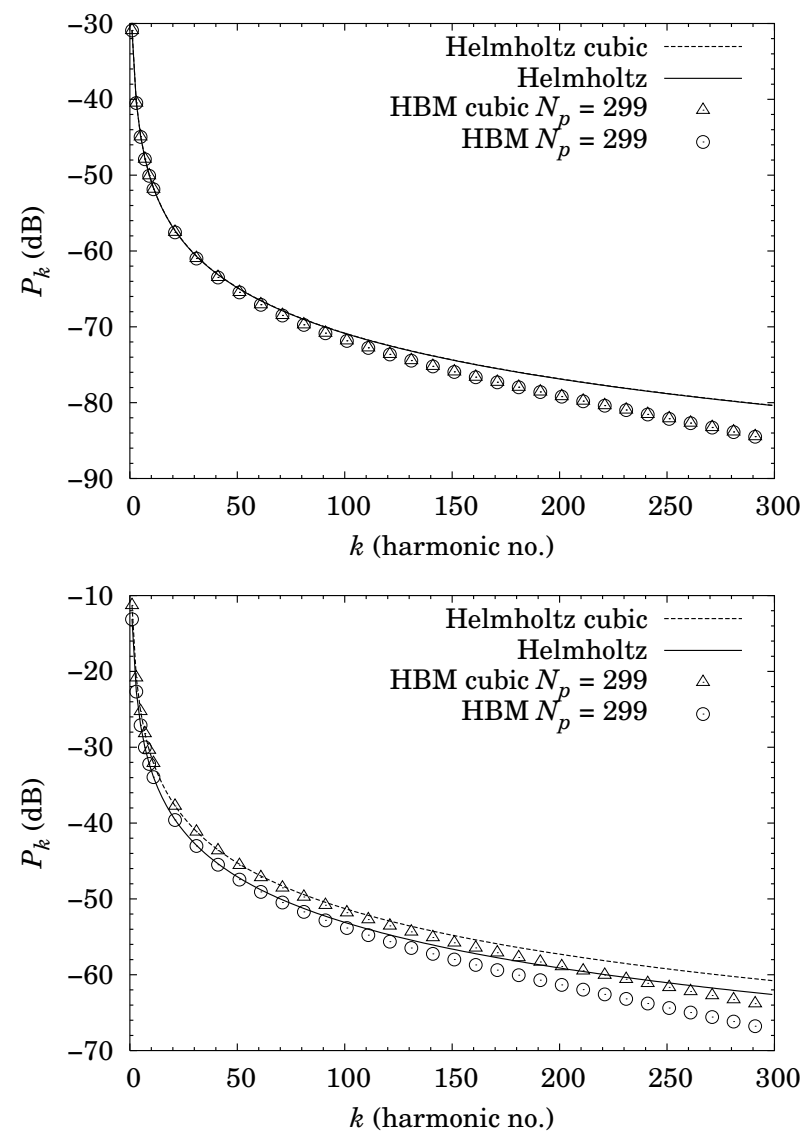

FIG. 10. The (lossless) Helmholtz motion and the (almost lossless) HBM for 299 harmonics using the full nonlinearity (23) and the cubic expansion (25) (a) close to the oscillation threshold $(\gamma=0.334)$ and $(\mathrm{b})$ far from it $(\gamma=0.40)$ for $\zeta=0.5, \eta=10^{-5}$. Above the 11th harmonic only every 10th harmonic is shown.

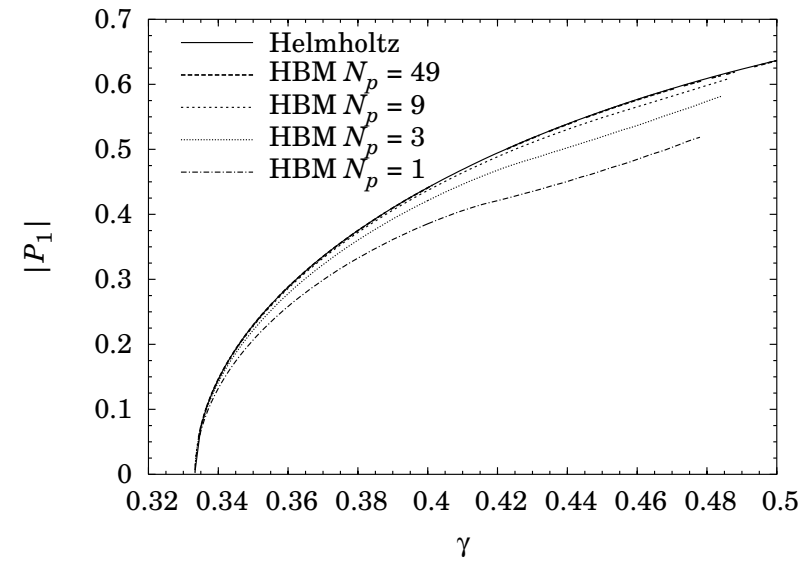

FIG. 11. Amplitude of first harmonic as the blowing pressure increases for the Helmholtz solution (24) and the HBM truncated to $1,3,9$, and 49 harmonics, the last coinciding with Helmholtz $\left(\zeta=0.5, \eta=10^{-5}\right)$

was used to make Harmbal calculate solution for each of a large number of subsequent values of $\gamma$ down to the oscillation threshold by using the previous solution as initial value. The procedure was repeated from $\gamma=0.4$ up to the point where the reed started to beat, i.e. for $p<\gamma-1$ in equation (23). Without losses (Helmholtz solution) the beating threshold does not arrive before $\gamma=0.5$, and this should be expected for the nearly lossless case studied with the HBM also. However, the number of harmonics $N_{p}$ taken into account in the HBM calculations is too small to follow the sharp edges of the square signal. The resulting overshoots in $p(t)$, as seen in Figure $8 \mathrm{~b}$, cause $p$ to prematurely exceed the criterion for beating. The beating threshold converges to 0.5 as $N_{p}$ increases (see also Ref.? ). Note that, for the chosen value of $\zeta$, it can be calculated following Hirschberg? (eq.(45)) that above $\gamma \simeq 0.45$, the Helmholtz solution loses its stability through a subharmonic bifurcation (a period-doubling occuring).

By Figure 11 we can also verify that the model experiences a direct Hopf bifurcation (which is known since the work of Grand et al.).? Thus, a single harmonic is enough to study the solution around the threshold. Far from the threshold, more harmonics have to be taken into account for $P_{1}$ to converge toward the Helmholtz solution. This is not obvious and for example contradictory with the hypothesis made for the VTM. ${ }^{\text {? }}$ Thus Harmbal appears as an interesting tool to evaluate the relevance of approximate methods according to the parameter values.

\section{Helmholtz oscillation for a stepped conical tube}

The saxophone works similarly to the clarinet, but the bore has a conic form. In this section we compare the HBM calculations with analytical results, and in order to calculate the Helmholtz motion when losses are ignored, we need to simplify the cone by assuming that it consists 
of a sequence of $N$ sylinders of length $l$ and cross section $S_{i}=\frac{1}{2} i(i+1) S_{1}, S_{1}=S$ being the cross section of the smallest cylinder, and $i=1, \ldots, N$ (see Ref.? ). The total length of the instrument is thus $L=N l$. The input impedance of such a stepped cone may be written as

$$
Z_{r}(\omega)=\frac{2 i}{\cot \left(\frac{\omega^{\prime}}{4}-i \alpha\left(\omega^{\prime}\right)\right)+\cot \left(\frac{\omega^{\prime}}{4 N}-i \alpha\left(\frac{\omega^{\prime}}{N}\right)\right)},
$$

where $\omega^{\prime} \triangleq 2 \omega /(N+1)$ when $\omega=2 \pi \hat{f} / f_{r}$, where $f_{r}$ is the first eigenfrequency of this resonator. We have ignored the dispersion term here. Equation (27) is used instead of equation (20), and the damping $\alpha(\omega)=\psi \eta \sqrt{\omega / 2 \pi}$ is zero in the analytic Helmholtz case and very small $\left(\eta=2 \cdot 10^{-5}\right.$ below which convergence became difficult) for the calculations with the HBM.

As before, the pressure amplitude of the ideal lossless case is calculated by solving $u(p)=u(-N p)$, and two solutions are possible: ${ }^{3}$

$$
\begin{aligned}
p^{ \pm}(\gamma) & =\frac{(N-1)(2-3 \gamma)}{2\left(N^{2}-N+1\right)} \\
& \pm \frac{\sqrt{(N-1)^{2}+(N+1)^{2}\left(-3 \gamma^{2}+4 \gamma-1\right)}}{2\left(N^{2}-N+1\right)}
\end{aligned}
$$

as long as $\gamma<1 /(N+1)$ for the standard Helmholtz motion $\left(p^{+}\right)$and $\gamma<N /(N+1)$ for the inverted one $\left(p^{-}\right)$, which is unstable. Above these limits $p^{+}=\gamma$ and $p^{-}=-\gamma / N$. The magnitude of the first harmonic of a square or rectangular wave is then given by

$$
P_{1}^{ \pm}(\gamma)=\frac{\sin \frac{\pi}{N+1}}{\frac{\pi}{N+1}} p^{ \pm}(\gamma)
$$

For $N=1$, equation (28) reduces to equation (24). For higher $N$, the pressure oscillation becomes asymmetric.

We take the case $N=2$ and get

$$
p^{ \pm}(\gamma)=\frac{1}{6}\left(2-3 \gamma \pm \sqrt{-27 \gamma^{2}+36 \gamma-8}\right) .
$$

This result is compared with HBM calculations in Figure 12 for $\gamma=0.31$. Theoretically, the spectrum of the Helmholtz solution, Figure 12a, shows that every third component is missing (actually zero) while the remaining components decrease in magnitude thus forming the asymmetric pressure oscillation as shown in Figure 12. The HBM, on the other hand, suggests that the first component in each pair be smaller than the second component. This results in a dip at the middle of the long, positive part of the period (i.e. on both extremities $t=0$ and $t=1024$ of the curve in Figure 12). The same was observed for $N=3$ and $N=4$, where the long part of the period was divided by similar dips into three and four parts, respectively (not shown). The number of time samples, $N_{t}$ did not change this fact, but as Figure 12 indicates, the dips gradually become narrower as the number of harmonics $N_{p}$ increases. This indicates
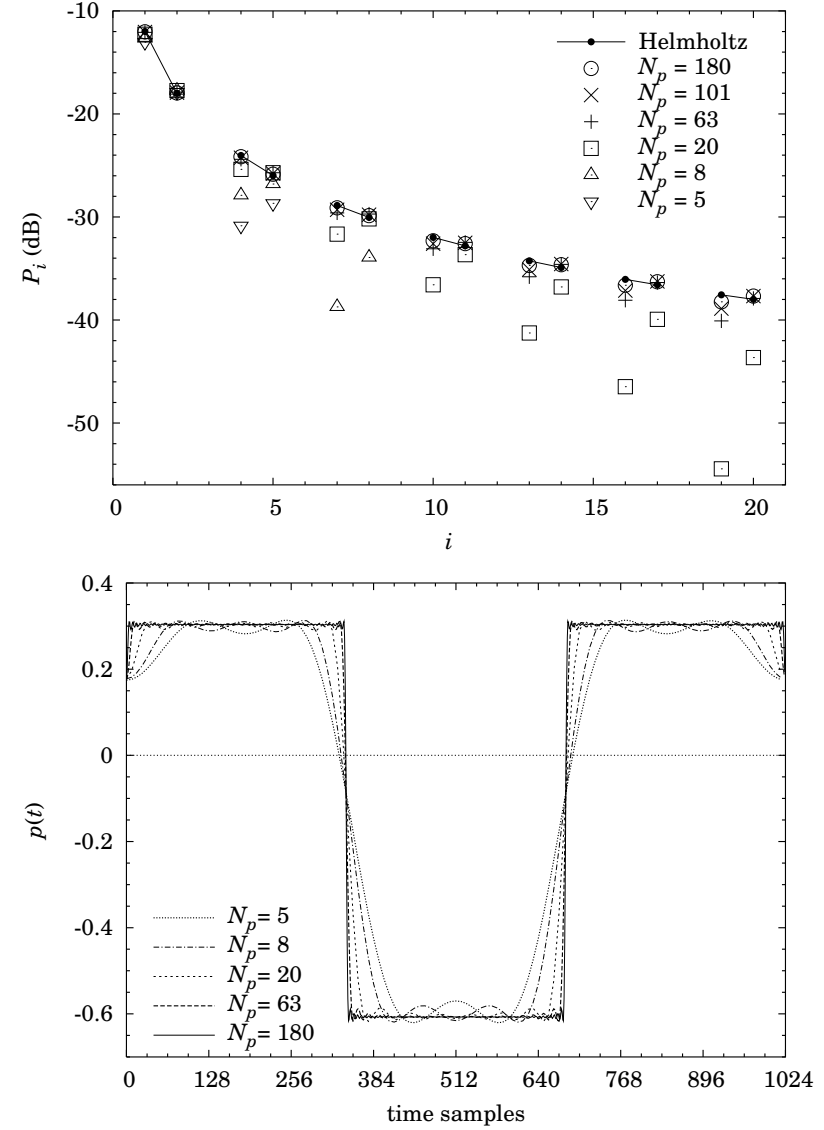

FIG. 12. Comparison between the standartd Helmholtz motion of a stepped cone $(N=2)$ and the HBM for various $N_{p}$ at $\gamma=0.31, \zeta=0.2$, and $\eta=2 \cdot 10^{-5}$. (a) The magnitude of the harmonics and (b) one oscillation period. $N_{t}$ varies from 128 for $N_{p}=5$ to 1024 for $N_{p}=180$.

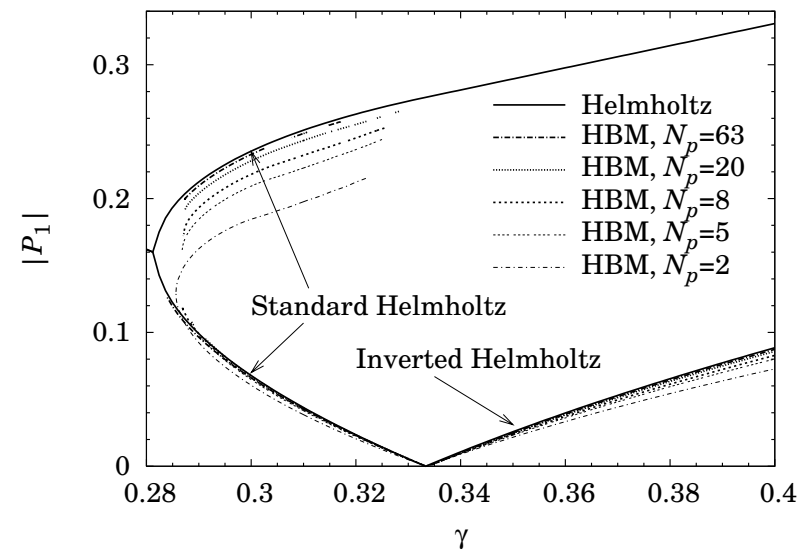

FIG. 13. Amplitude of first harmonic $P_{1}$ as a function of the blowing pressure $\gamma$ for the Helmholtz solution (30) for 2-stepped cone and the HBM truncated to 2, 5, ., and 63 harmonics, the last coinciding with Helmholtz $(\zeta=0.2, \eta=$ $\left.2 \cdot 10^{-5}\right)$. Only nonbeating regimes are shown. 
that the HBM approaches the Helmholtz solution as $N_{p}$ approaches infinity.

A bifurcation diagram is plotted in Figure [13] Similarly to Figure 11 for the cylindrical bore, the amplitude of the first harmonic is plotted for different number of harmonics as a function of the blowing pressure $\gamma$. The Helmholtz solution (equation (29) with $N=2$ ) is also plotted. As shown by Ollivier et al., ? the lower part of the upper branch and the branch of the inverted Helmholtz motion are unstable.

In practice, these curves are more difficult to obtain with hbmap than for the cylindrical bore, especially close to the subcritical oscillation threshold around $\gamma=0.28$, where computation was not possible at this low losses. More sophisticated continuation schemes should be considered to obtain complete curves. However, it is obvious from the diagram that the model experiences a subcritical Hopf bifurcation, which agrees with the conclusion of Grand et al..? This means that a single-harmonic approximation is not enough to study the solution around this threshold, since the small-amplitude hypothesis does not hold. Further from the threshold, convergence toward the Helmholtz motion is ensured as the number of harmonics $N_{p}$ is increased.

Only the nonbeating reed regime is considered in the figure and, similarly to Figure [1] it can be noted that the beating threshold for the model with $N_{p}$ harmonics depends on $N_{p}$ but converges toward the Helmholtz threshold $\gamma=1 / 3$ (corresponding to the lossless, continuous system) as $N_{p}$ is increased.

\section{Validation with time-domain model}

When adding a mass and damping to the reed or viscous losses and dispersion to the pipe, it is more difficult to compare Harmbal results with analytic solutions. This has been done by Fritz et al. ${ }^{?}$ as far as the playing frequency is concerned, by comparison with approximate analytical formula. Here, we propose to confront both the playing frequency and the amplitude of the first partial with numerical results obtained with a timedomain method. We use a newly developed (real-time) time-domain method (here called TDM) by Guillemain et al.. ${ }^{\text {? }}$ It is based on the same set of equations as presented in Section IVA except that the impedance of the bore is slightly modified to be expressed as an infinite impulse response. In the Fourier domain, it can be expressed as

$$
Z_{r}(\tilde{\omega})=\frac{1-a_{1} e^{-i \tilde{\omega}}-b_{0} e^{-i \tilde{\omega} D}}{1-a_{1} e^{-i \tilde{\omega}}+b_{0} e^{-i \tilde{\omega} D}}
$$

where $\tilde{\omega}=\hat{\omega} / f_{s}, f_{s}$ being the sampling frequency, and the integer $D=\operatorname{round}\left(f_{s} / 2 f_{r}\right)$ the time delay in samples for the sound wave to propagate to the end of the bore and back. The constants $a_{1}$ and $b_{0}$ are to be adjusted so that the two first peaks of resonance have the same amplitude as the two first peaks of equation (20).

To express equation (31) using our terminology, we
TABLE I. The values of $M$ and $R$ for three strengths of reed interaction. The bore parameters are $D=247\left(f_{r}=\right.$ $103.4 \mathrm{~Hz}), a_{1}=0.899$, and $b_{0}=0.0946$ for sampling frequency $f_{s}=51100 \mathrm{~Hz}$.

\begin{tabular}{l|rccc}
\hline Reed & $\omega_{e} / \mathrm{Hz}$ & $q_{e}$ & $M$ & $R$ \\
\hline Weak & 10000 & 0.1 & $1.070 \cdot 10^{-4}$ & $1.034 \cdot 10^{-3}$ \\
Normal & 2500 & 0.2 & $1.712 \cdot 10^{-3}$ & $8.28 \cdot 10^{-3}$ \\
\hline
\end{tabular}

remember that $\omega=2 \pi \hat{f} / f_{r}$ and obtain

$$
Z_{r}(\omega)=\frac{1-a_{1} e^{-i \omega \frac{f_{r}}{f_{s}}}-b_{0} e^{-i \omega / 2}}{1-a_{1} e^{-i \omega \frac{f_{r}}{f_{s}}}+b_{0} e^{-i \omega / 2}} .
$$

In this section, we also include the mass and damping of the reed, so $M$ and $R$ are no longer zero. The TDM does not work for $M=R=0$, or even for values close to this, so we have used a reed with weak interaction with the pipe resonance as well as one with close to normal reed impedance. The corresponding values for $\omega_{e}$ and $q_{e} \triangleq g_{e} / \omega_{e}$ are shown in Table [1] Figure 14] shows the bifurcation diagram for two values of $\zeta$ and for weak and normal reed impedance, while Figure 14 s shows the corresponding variation in the dimensionless playing frequency $f_{p} / f_{r}$. The lines represent the continuous solutions of the HBM, and the symbols show a set of results derived from the steady-state part of the TDM signal. The TDM symbols fall well on the lines of the HBM, except for $\zeta=0.50$ when $\gamma$ approaches 0.5. Then the TDM experiences period doubling, i.e. two subsequent periods of the signal differ. At the same time, not being able to show subharmonics, the HBM shows signs of a beating reed, possibly a solution that is unstable and thus not attainable by time-domain methods.

Note that three points have to be verified before comparing results from the HBM and the TDM:

The numerical scheme used in the TDM to approximate the time derivatives in the reed equation (15) requires discretization. Depending on the sampling frequency $f_{s}$, the peak of resonance of the reed deviates more or less from the one given by the continuous equation. For normal reed interaction $\left(f_{e}=2500 \mathrm{~Hz}\right)$, the deviation is negligible, but it may become significant in the case of weak reed interaction, where the peak is at $10000 \mathrm{~Hz}$. However, the fact that the reed and the bore interact weakly in the latter case, implies that the exact position of the peak has little importance. Therefore, at the used sampling frequency, the discretization in the TDM is not compensated for in the HBM calculations.

Then there should be agreement between the sampling frequency $f_{s}$ in the TDM and the number of samples $N_{t}$ per period in the HBM. Their relation is given by

$$
N_{t}=\frac{f_{s}}{f_{p}} .
$$

In order to have a sufficiently high sampling rate, we have chosen $N_{t}=512$. The playing frequency $f_{p}$ is plotted in 

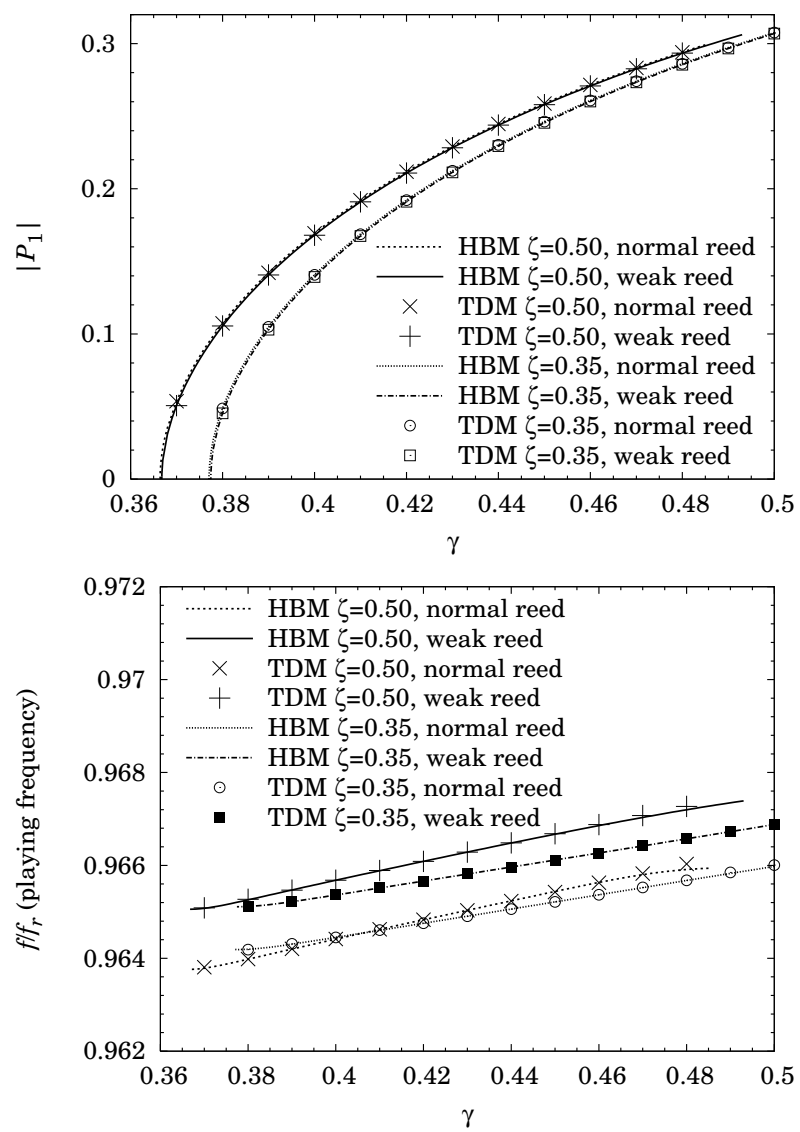

FIG. 14. Comparison between HBM and TDM of the amplitude of (a) the first harmonic $P_{1}$ and (b) the dimensionless playing frequency $f_{p} / f_{r}$ as the blowing pressure $\gamma$ increases for a clarinet-like system with viscous losses and weak and normal reed interaction. TDM values for $\zeta=0.50$ and $\gamma>0.48$ are omitted due to period doubling. So are the beating regimes of HBM calculations. $\left(f_{s}=51100 \mathrm{~Hz}, N_{t}=512, f_{r}=103.4 \mathrm{~Hz}\right.$, $\left.N_{p}=15\right)$

Figure 14], and we used an average $f_{s}=51100 \mathrm{~Hz}$ for both the HBM and the TDM.

Finally, it seems also necessary that $N_{p}$ and $N_{t}$ are chosen so that

$$
N_{p}+1=\frac{N_{t}}{2}
$$

In practice, however, when comparing bifurcation diagrams of the first harmonic $P_{1}$, as in Figure 14 rather low values of $N_{p}$ give good results. Nevertheless, more harmonics are obviously needed to compare waveforms in the time domain, especially far from the oscillation threshold.

\section{PRACTICAL EXPERIENCES}

\section{A. Multiple solutions}

As we consider a nonlinear problem, we cannot anticipate the number of solutions. Therefore, it should not be
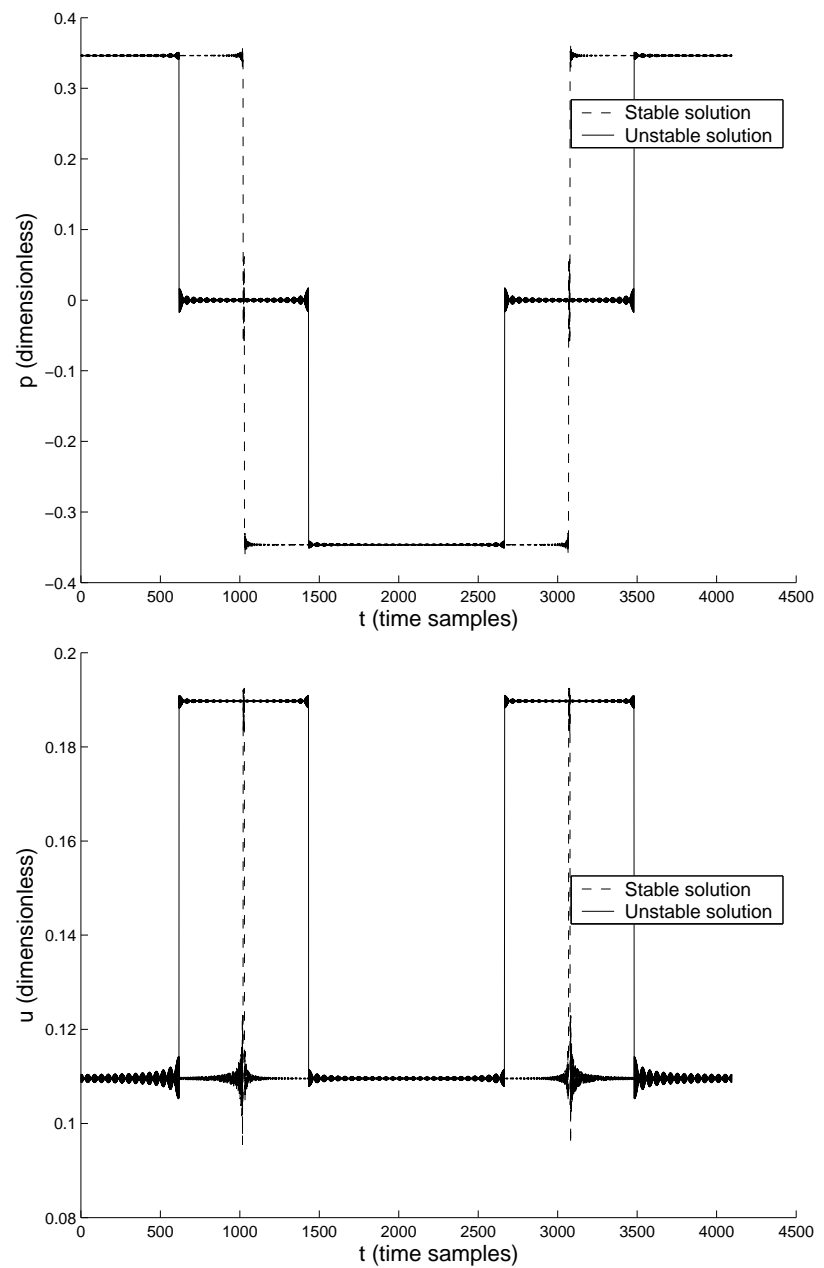

FIG. 15. The pressure (a) and volume-flow (b) wave form of the Helmholtz solution and a 3-level sister solution calculated by the HBM employing the simple clarinet model with $\zeta=$ $0.5, \gamma=0.4, N_{p}=99, \eta=10^{-5}$.

surprising that it is possible to obtain multiple solutions for a given set of parameter values. When searching for a particular solution, this may be a practical problem. Fritz et al. ${ }^{?}$ have discovered that some solutions seem to disappear when increasing the number of harmonics $N_{p}$, implying that solutions may arise from the truncation to a finite $N_{p}$. We have now discovered alternative solutions that persist even at very high $N_{p}$.

Let us illustrate this with the simple model of the clarinet used in Section IVB 1 where the reed is a spring without mass or damping, the nonlinearity is given by equation (23), and the bore is an ideal cylinder with nearly lossless propagation and no dispersion. Figure 15 shows a three-level sister solution together with the related Helmholtz solution for a large number of harmonics, $N_{p}=2000$.

A solution of the lossless problem should satisfy the 
criteria?

$$
\left\{\begin{array}{l}
p(t+\pi)=-p(t) \\
u(t+\pi)=u(t)
\end{array}\right.
$$

(the dimensionless period being $2 \pi$ ), as well as the conditions stated before equation (24), noting that $p(t)=u(t)=0$ for all $t$ is the static solution. It is easily verified graphically that both of the solutions in Figure 15] satisfy these conditions. Moreover, since they also satisfy equation (23), the three-level solution is a solution of the lossless model.

Whereas the system of time-domain equations (35) has an infinity of solutions, truncation in frequency-domain limits the number of solutions. The unique solution of the HBM with only one harmonic is obviously a sine. Let us analyse the situation in the simplest nontrivial case of the lossless problem with two odd harmonics and a cubic expansion for nonlinear coupling. Ignoring even harmonics, the HBM gives a system of two equations (see Kergomard et al.)? :

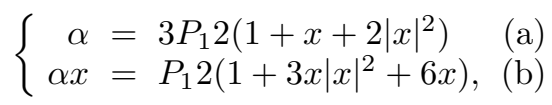

where $\alpha=-A / C$ and $x=P_{3} / P_{1}$. As equation (36 ) imposes $P_{3}$ to be real, solving this system amounts to solving

$$
x^{3}+x^{2}-x=1 / 3 .
$$

This equation has three real solutions $x \simeq-1.5151$, -0.2776 and 0.7926 . All of them are found by Harmbal for negligible losses $\left(\eta=10^{-5}\right)$, and the corresponding waveforms are presented in Figure [16] We note that the second solution leads to the Helmholtz motion when increasing the number of harmonics (with the theoretical value known to be $x=-1 / 3$ ) whereas the third one corresponds to the three-level solution in Figure 15] We can also easily imagine that these three solutions of the truncated problem are three-harmonic approximations of square waves that are distributed on three levels: $p^{ \pm} \simeq \pm 0.5$ and $p=0$. Respectively, they have two, zero, and one steps at the zero-level. It should be noted that the conditions (35) for the continuous problem do not constrain the duration of each step. Figure 17 shows two such twin solutions for $N_{p}=99$ corresponding to the three-level solution in Figure [16] This has to be kept in mind when increasing $N_{p}$ using the HBM.

While the Helmholtz motion is known to be stable, the two three-level solutions can be considered as a combination of the static solution (the zero level) and the square wave (two levels with opposite values). Since we know from Kergomard? that in the case of ideal propagation (neither losses nor dispersion), the stability domain of these two solutions are mutually exclusive, it can be concluded that the three-level solutions are unstable.

Taking into account losses in the propagation does not make the three-level solutions vanish. But a simple reasoning to determine the stability of this solution is not
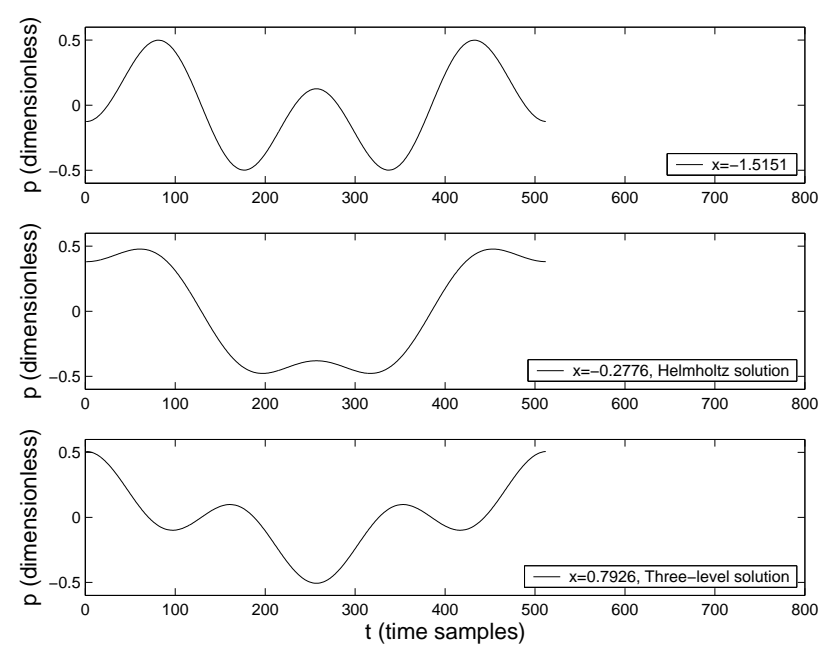

FIG. 16. The pressure waveform of the three solutions found by the HBM with $N_{p}=3$ employing the simple clarinet model with $\zeta=0.5, \gamma=0.4, \eta=10^{-5}$.

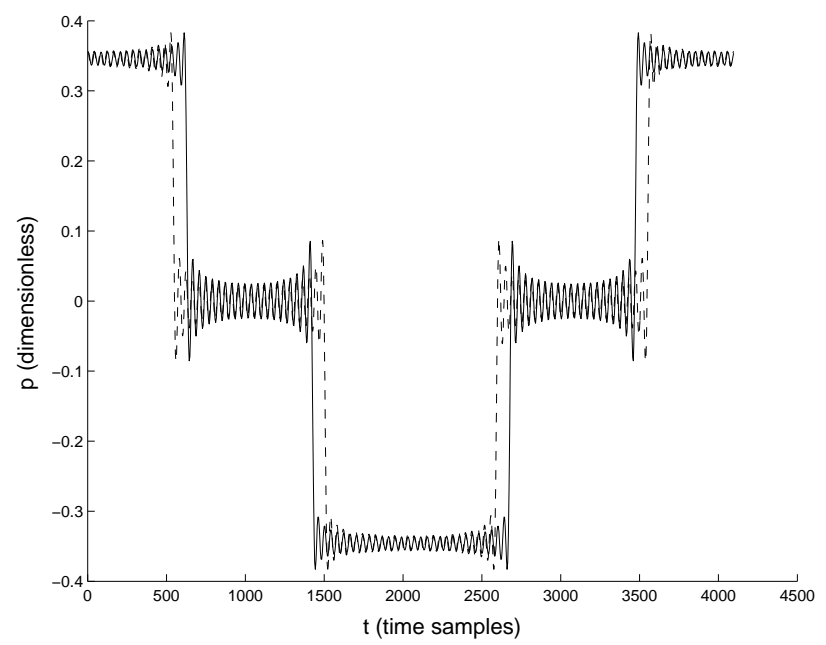

FIG. 17. The pressure waveform of two solutions that differ by the duration of their steps, found by the HBM employing the simple clarinet model with $\zeta=0.5, \gamma=0.4, \eta=10^{-5}$, $N_{p}=99$.

possible in this case. To the authors knowledge, however, such a solution has never been observed experimentally at low level of excitation.

\section{B. Initial value of the playing frequency}

A practical difficulty encountered is the convergence of the playing frequency $f_{p}$. If its initial value is not close enough to the solution, divergence is almost inevitable. This occurs because the resonator impedance $Z_{r}$ tends to vanish outside the immediate surroundings of the resonance peaks of the resonator, rendering $\vec{F}\left(\vec{P}, f_{p}\right)$ very small and thereby $\vec{G} \simeq \vec{P} / P_{1}$ nearly constant with respect to $f_{p}$. The slope $\partial \vec{G} / \partial f_{p}$ thus becomes close to 
zero, the Newton step leads far away from the solution, and convergence fails. Dissipation widens the resonance peaks and thus also the convergence range.

For a simple system where the playing frequency is known to correspond to a resonance peak of the tube, initializing $f_{p}$ is easy. However, with dispersion or other inharmonic effects, choosing an initial value for $f_{p}$ may be difficult. In Harmbal the problem may to some extent be avoided by the possibility of gradually adding the dispersion (or other inharmonic effects), so that the playing frequency can be followed quasi-continuously from a known solution without dispersion, for instance by using hbmap.

\section{CONCLUSIONS}

The harmonic balance method (HBM) is suited for studies of self-sustained oscillations of musical instruments, and the computer program Harmbal has been developed for this application. It is available with its source code, ${ }^{?}$ has a free licence, and is already in use by several researchers. It is programmed in $\mathrm{C}$, runs fast, and is easily used by other application, such as for continuation purposes.

Some difficulties are related to the digital sampling of the signal and can be solved by introducing a backtracking mechanism. When using a large number of harmonics, the extreme case of the (lossless) Helmholtz motion can be solved for different shapes of resonators. Nevertheless, the value of the first harmonic $P_{1}$ seems to be well predicted by lower values of $N_{p}$, in particular close to the threshold of a direct bifurcation. For the saxophone we used a stepped-cone bore and observed one or more dips during the longest part of the period, depend- ing on the number of steps. These dips approach pure impulses as $N_{p}$ increases. The number of samples $N_{t}$ in a period showed to be insignificant for these dips.

The HBM can lead to some alternative solutions for a unique set of parameters. The nondissipative versions of these solutions satisfy the continuous model equations, but they are not stable and thus cannot be attained by ab initio time-domain calculations. Another problem is the great sensitivity to the guessed playing frequency.

As a consequence, a certain expertise is needed in order to use the method, but, thanks to an automatic continuation procedure, the calculation is easy. We note that also experimental results can be used for the impedance of the resonator.

\section{ACKNOWLEDGMENTS}

The Europeen Union through the MOSART project is acknowledged for financial support. We would also like to thank Claudia Fritz at IRCAM in Paris for thorough testing and valuable feedback, Joël Gilbert at Laboratoire d'Acoustique de l'Université du Maine (LAUM) in Le Mans, and Philippe Guillemain at the Laboratoire de Mécanique et d'Acoustique at CNRS in Marseille for fruitful discussions during the work, and the latter also for kindly providing some Matlab code for the time-domain model.

${ }^{1}$ Self-sustained is a term indicating oscillation driven by a constant energy input.

${ }^{2}$ The term non-interactive means that the user has no influence on the program while it is running.

${ }^{3}$ This result corrects equation (14) in ref.? 